\section{Q-factor monitoring of optical signal-to-noise ratio degradation in optical DPSK transmission}

\author{
K.-C. Jong, H.-W. Tsao and S.-L. Lee
}

An asynchronous sampling monitor for optical DPSK signals by using a two-tap histogram is proposed. By means of statistical analysis, a parameter is defined to measure the Q-factor to indicate the OSNR degradation. Compared to traditional monitoring methods, this method requires no synchronisation before sampling and is thus cost-effective.

Introduction: Currently in long-haul fibre transmission, the optical phase-shift keying (PSK) format has attracted attention mainly because it has better receiver sensitivity than the conventional on-off keying (OOK) format [1]. Hence, the differential PSK (DPSK) format is widely adopted in high speed photonic networks. However, to diagnose any system impairment immediately, the monitoring of its signal quality is an essential issue. Previously, synchronous sampling for Q-factor monitoring has been proposed [2,3], but it requires clock recovery. To solve this problem, an asynchronous sampling without requiring any synchronisation technique is preferred [4]. Moreover, Dods and Anderson use two-tap plots generated by asynchronous delay-tap sampling to monitor the wave distortion of OOK signals [5]. By analysing the pattern of the two-tap histograms, system impairments such as optical signal-to-noise ratio (OSNR) degradation and chromatic dispersion can be investigated.

In this Letter, we present a novel asynchronous sampling technique to monitor DPSK signals from two-tap histograms, which can be obtained naturally from the two output ports of an optical DPSK demodulator. This method avoids use of another splitter and delay line to perform the task. The signal statistics obtained at the two output ports directly indicate the signal quality to the balanced receivers for detecting the DPSK signals. With this scheme, a low cost Q-factor monitoring for DPSK signals can be realised without clock recovery electronics

Proposed method: A simple block diagram for our method is shown in Fig. 1. A laser light of $1550 \mathrm{~nm}$ wavelength is phase modulated by a $10 \mathrm{Gbit} / \mathrm{s}$ differential-encoded pseudorandom bit sequence (PRBS). The fibre link consists of five spans of a $50 \mathrm{~km}$ singlemode fibre (SMF), a $10 \mathrm{~km}$ dispersion-compensation fibre (DCF) and two erbium-doped fibre amplifiers (EDFAs). A delay interferometer (DI) is used for phase demodulation. The signal at one of the output ports represents the constructive interference of the two DI arms, while the other port indicates the destructive interference result. Hence, these two ports have logically conjugated information. To change the signal OSNR, a tunable attenuator is placed in front of the DI. Two independent photoreceivers are connected to the two output ports, and a time difference $\Delta \tau$ is added to indicate possible relative propagation delay between the two signal paths. The detected voltage at each output port is sampled by separate analogue-to-digital converters (ADCs) driven with the same clock, which is not synchronous with the data rate. We use the sampled voltages from both the constructive and destructive interference port as a pair for statistical analysis. The rearranged sample pairs can be plotted as a three-dimensional histogram. Fig. 2 shows a typical two-tap histogram for the system shown in Fig. 1 where the actual Q-factor $\left(Q_{t}\right)$ is $18.07 \mathrm{~dB}$ and $\Delta \tau$ is $20 \mathrm{ps}$. The two peaks that appear in the histogram correspond to the phase change 'zero' and ' $\pi$ ' between adjacent bits of the differential encoder. Considering the finite bandwidth of the photoreceivers, the sampled voltage contains the rising or falling edge of the waveform that appears in the middle region between two peaks (MRTP).

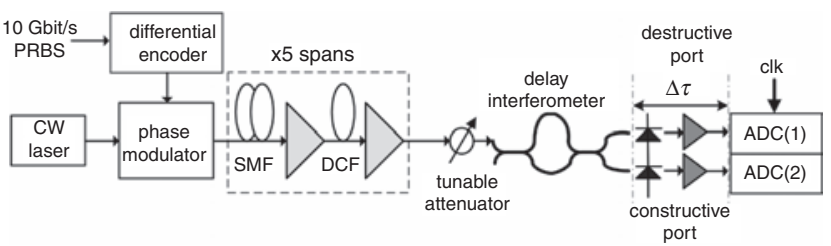

Fig. 1 Block diagram of simulated system setup

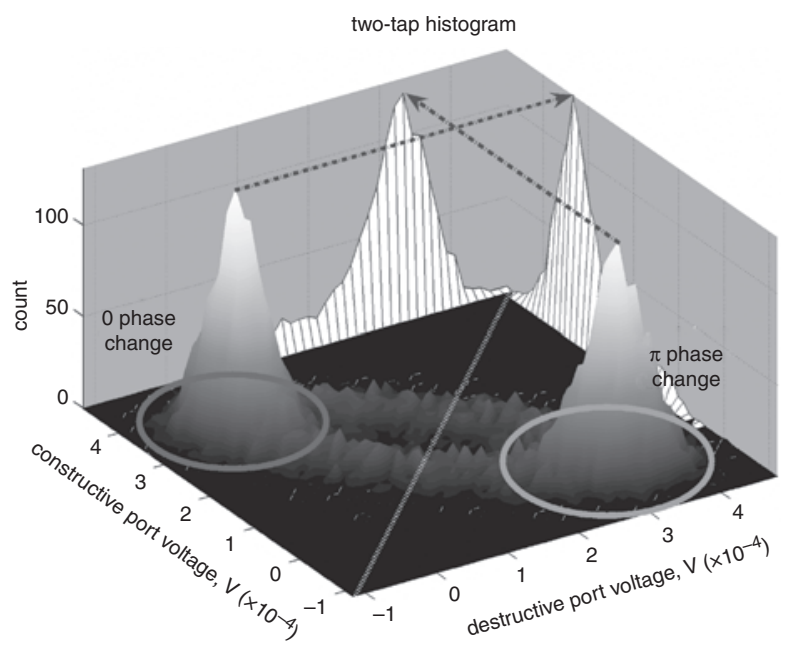

Fig. 2 Two-tap histogram for signal with $Q_{t}=16.5 \mathrm{~dB}$ and $\Delta \tau=20 \mathrm{ps}$

One of the advantages of using the two-tap histogram is that the dispersion-related distortion can be resolved. For example, the chromatic dispersion that leads to FM-to-AM conversion can cause amplitude distortion to the DPSK signals and change the histogram [6]. In this Letter we focus on the case of OSNR degradation and the analysis of the other distortion effects by the proposed scheme will be addressed elsewhere.

To capture signal quality information from this histogram, we project the two peaks individually on a different co-ordinate axis. The statistical parameters of each projection can then be estimated by Gaussian curve fitting. A new parameter $Q_{2 T}$ is defined as follows to identify the signal quality:

$$
Q_{2 T}=20 \times \log \frac{\sqrt{{\overline{V_{0}}}^{2}+{\overline{V_{\pi}}}^{2}}}{\sigma_{0}+\sigma_{\pi}}
$$

where $\overline{V_{0}}$ and $\overline{V_{\pi}}$ stand for the mean value of the fitted 'zero' and ' $\pi$ ' statistics, respectively. Similarly, $\sigma_{0}$ and $\sigma_{\pi}$ are their standard deviations, respectively.
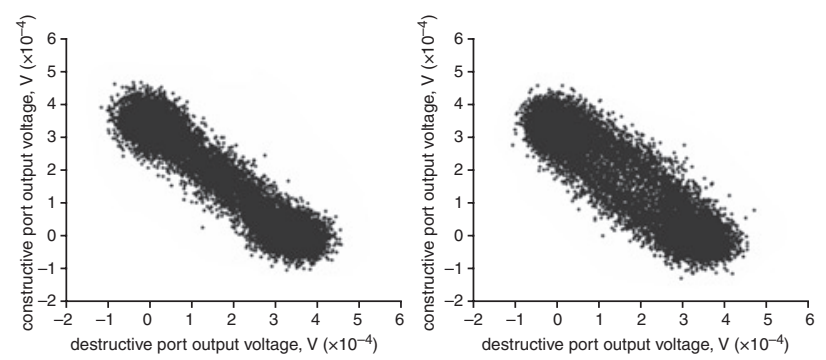

a

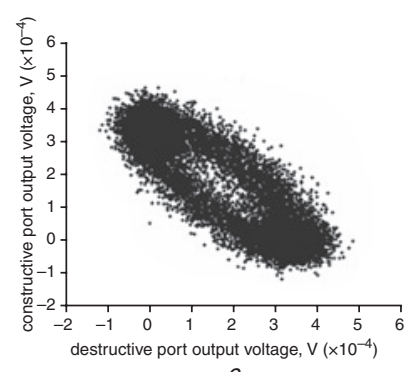

$c$

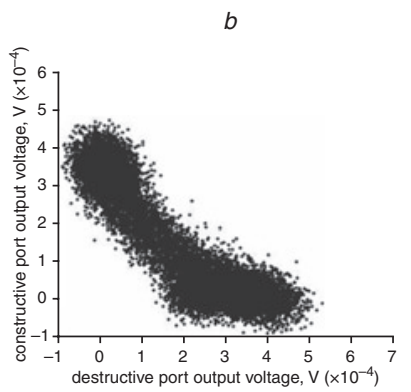

d

Fig. 3 Two-tap histograms for different $\Delta \tau$ and dispersion with $Q_{t} \simeq$ $16.5 \mathrm{~dB}$

a $\Delta \tau=0 \mathrm{ps}$, no dispersion

$b \Delta \tau=10 \mathrm{ps}$, no dispersion

$c \Delta \tau=20 \mathrm{ps}$, no dispersion

$d \Delta \tau=0 \mathrm{ps}$, transmission over $25 \mathrm{~km}$ uncompensated SMF

Simulation results: Fig. 3 compares the two-tap histogram for different $\Delta \tau$; each resembles an oblique eye pattern. When $\Delta \tau=0$, the histogram becomes a line because the samples of two DI outputs are logically conjugated, as shown in Fig. $3 a$. With a nonzero time-delay $|\Delta \tau|$ between the two monitoring paths, the histogram becomes an elliptic shape in 
the MRTP region, as shown in Figs. $3 b, c$. Although the MRTP distribution of the two-tap histogram depends on the value of $|\Delta \tau|$, the mean and standard deviation for the two peaks are almost constant after curve fitting. Because the sample count of the MRTP region is sufficiently low as $|\Delta \tau|$ is smaller than $20 \mathrm{ps}$, it does not play an important role in the OSNR measurement. When the signal transmission is subject to the dispersion effect, the histogram is distorted, as shown in Fig. $3 d$ for a transmission over a $25 \mathrm{~km}$ uncompensated singlemode fibre. This result indicates that this scheme can be used to detect the dispersion for DPSK signals.

For the purpose of $Q_{t}$ estimation, it is essential to find the relationship between $Q_{t}$ and $Q_{2 T}$. Fig. 4 shows the dependence of $Q_{2 T}$ on $Q_{t}$ as the value of $\Delta \tau$ is changed. As long as $|\Delta \tau| \leqq 20 \mathrm{ps}$, the monitored parameter is insensitive to the value of $\Delta \tau$, so the relationship between $Q_{t}$ and $Q_{2 T}$ is nearly identical, i.e. we can adopt two individual photoreceivers without precisely temporal match and still get a reproducible $Q_{t}$. Furthermore, the proposed scheme uses the asynchronous sampling technique, hence there is no need of any timing extraction equipment. By this method, the advantages of low cost and low complexity can be obtained.

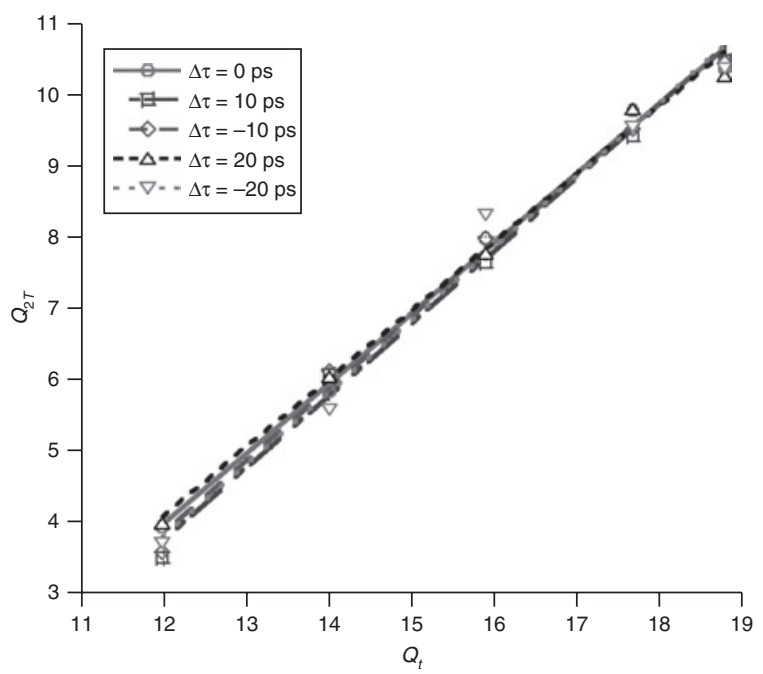

Fig. 4 Relationship between $Q_{2 T}$ and $Q_{t}$
Conclusion: We present a novel asynchronous monitoring technique for optical DPSK transmission, based on the property of the DI output ports and the use of a two-tap histogram. Simulation results show that the fitting parameters are insensitive to the temporal-mismatch between two individual photoreceivers. With this method, a low cost and less complicated Q-factor monitoring system can be realised. It can also be used for detecting dispersion effects.

(C) The Institution of Engineering and Technology 2008

18 February 2008

Electronics Letters online no: 20080443

doi: 10.1049/el:20080443

K.-C. Jong and H.-W. Tsao (Graduate Institute of Photonics and Optoelectronics and Department of Electrical Engineering, National Taiwan University, 1, Sec. 4, Roosevelt Road, Taipei 10617, Taiwan, Republic of China)

E-mail: tsaohw@cc.ee.ntu.edu.tw

S.-L. Lee (Department of Electronic Engineering, National Taiwan University of Science and Technology, 43, Sec. 4, Keelung Rd., Taipei 10607, Taiwan, Republic of China)

\section{References}

1 Gnauck, A.H., and Winzer, P.J.: 'Optical phase-shift-keyed transmission', J. Lightwave Technol., 2005, 23, (1), pp. 115-130

2 Bergano, N.S., Kerfoot, F.W., and Davidson, C.R.: 'Margin measurements in optical amplifier systems', IEEE Photonics Technol. Lett., 1993, 5, (3), pp. 304-306

3 Agrawal, G.P.: 'Lightwave technology: telecommunication systems' (Wiley, Hoboken, NJ, 2005)

4 Shake, I., and Takara, H.: 'Averaged Q-factor method using amplitude histogram evaluation for transparent monitoring of optical signal-to-noise ratio degradation in optical transmission system', J. Lightwave Technol., 2002, 20, (8), pp. 1367-1373

5 Dods, S.D., and Anderson, T.B.: 'Optical performance monitoring technique using delay tap asynchronous waveform sampling'. Proc. Optical Fiber Conf., Anaheim, CA, USA, 2006 (paper OThP5)

6 Chraplyvy, A.R., Tkach, R.W., Buhl, L.L., and Alferness, R.C.: 'Phase modulation to amplitude modulation conversion of $\mathrm{CW}$ laser light in optical fibres', Electron. Lett., 1986, 22, (8), pp. 409-411 